\section{New front against TNF}

\section{By Lauren Martz, Staff Writer}

New York University School of Medicine researchers have designed a molecule based on the growth factor progranulin that could help treat rheumatoid arthritis and other autoimmune diseases. ${ }^{1}$ The team has formed a company, ATreaon, to develop the compound and related analogs.

Previous work has suggested roles for progranulin (PGRN) in cancer, ${ }^{2}$ inflammation, ${ }^{3}$ host defense, ${ }^{4}$ cartilage development and degeneration ${ }^{5}$ and neurological functions. ${ }^{6,7}$ However, using or targeting the molecule has been difficult because its receptors remained unknown.

Now, Chuan-ju Liu and colleagues at NYU have found that PGRN binds tumor necrosis factor receptors (TNFRs) and blocks their interactions with tumor necrosis factor- $\alpha$ (TNF- $\alpha$ ), thus halting inflammatory signaling.

Liu is an associate professor at the NYU School of Medicine.

In a yeast two-hybrid screen using PGRN as bait, the team identified tumor necrosis factor receptor 1 (TNFRSF1A; TNFR1; CD120a) and TNFR2 as the main binding proteins for PGRN. The group confirmed the association through coimmunoprecipitation experiments in human chondrocytes.

In a mouse model of collagen-induced arthritis, knocking out Pgrn caused the development of more severe arthritis with bone and joint destruction, more rapid disease onset, greater disease incidence and higher levels of bone-resorbing osteoclasts than those in wild-type littermate controls.

The group also showed that administration of recombinant human PGRN (rhPGRN) to the knockouts blocked disease progression and prevented symptoms of inflammatory arthritis including synovitis, pannus formation, tissue destruction and loss of cartilage compared with administration of saline.

The findings were replicated in a mouse model of inflammatory arthritis induced by transgenic expression of TNF- $\alpha$.

With the help of Cytovance Biologics LLC, a contract biologics manufacturer, the NYU team developed a molecule called Atsttrin (Antagonist of TNF/TNFR Signaling via Targeting to TNF Receptors), which includes parts of three granulin (GRN) domains from progranulin as well as three of the protein's linker regions. Despite its smaller size, Atsttrin retains the functionality of PGRN on TNF receptors.
Compared with the parent molecule, Atsttrin also was more selective for the two TNFRs than for other closely related receptors.

Atsttrin also had higher affinity for TNFR2 than TNFR1-a finding that Martin Bachmann, CSO and EVP at Cytos Biotechnology AG, said needs to be further probed. "The fact that the molecule has a higher affinity for TNFR2 than TNFR1 may change its therapeutic properties compared with mAbs against TNF," he said. "This may or may not be beneficial."

Cytos' CYT020-TNFQb, a vaccine that binds TNF- $\alpha$ and was developed with the company's Immunodrug platform, is in preclinical testing to treat inflammation.

In the collagen-induced arthritis mouse model, Atsttrin decreased disease severity and delayed onset and progression better than rhPGRN; in a collagen antibody-induced arthritis mouse model, Atsttrin was more effective at treating inflammation than both rhPGRN and Enbrel etanercept, a soluble TNF receptor marketed by Amgen Inc., Pfizer Inc. and Takeda Pharmaceutical Co. Ltd. for RA and a variety of other autoimmune indications.

Atsttrin was well absorbed via intraperitoneal administration and had high stability and a half-life of about five days.

The findings were published in Science. The paper also included researchers from Shandong University School of Medicine, Weill Cornell Medical College, Yale School of Medicine, Nankai University and Texas A\&M Health Science Center.

In the fall of 2010, Jeffrey Su and colleagues formed ATreaon to develop Atsttrin and related compounds.

$\mathrm{Su}$ is $\mathrm{CSO}$ at Cytovance, which has not invested in the new compound but is a potential service provider to make the molecule.

ATreaon has raised an undisclosed amount of seed money and used the funds to license Atsttrin from the university and to develop analogs. The company hopes to put its lead compound into the clinic in the next 12 months for an autoimmune indication and is in talks with VCs and private equity investors to secure funding for INDenabling studies, GMP manufacturing and clinical studies.

Liu told SciBX that Atsttrin could be developed for multiple TNF-associated conditions and pathologies including systemic lupus erythematosus (SLE), inflammatory bowel disease (IBD), ankylosing spondylitis, plaque psoriasis and psoriatic arthritis.

\section{Room in the sandbox}

Although the market for TNF modulators is crowded-there are five marketed biologics that block TNF-ATreaon thinks its product initially could be used behind those drugs or in the about $50 \%$ of RA patients who do not respond to them.

"The unique anti-inflammatory mechanism of action of Atsttrin [means it] may be effective for those patients who do not respond to current TNF inhibitor treatments," said Su.

"It will also be quite interesting to find out whether this strategy 
can be used as an early intervention to prevent progression of symptoms or whether it works as an agent to use once other options have failed. I could imagine Atsttrin having both uses," said Andrew Bateman, a professor in the Department of Medicine at McGill University. "It will also be interesting to find out whether Atsttrin can be used along with conventional agents in combination therapy."

Bateman's research on the role of PGRN in wound healing showed that the protein promotes repair and regeneration of damaged tissues. "One could imagine that progranulin or an analog could repair the tissue damaged during the course of disease once the inflammation is under control," he said. "TNF has a lot of effects on immune responses and is a major switch for inflammation. TNF is not a bad thing, but it is a problem when it gets out of control and can be very dangerous. Rather than squishing it completely and blocking its beneficial functions, the goal is to be able to bring it back to a healthy range. It will be interesting to see if progranulin or related analogs can bring it back down to appropriate levels. This will take a lot of tuning to get the conditions right."

In terms of safety, Aihao Ding, professor in the Department of Microbiology and Immunology at Weill Cornell, thinks Atsttrin could enjoy an advantage over anti-TNF antibodies.

"One assumes Atsttrin would have fewer side effects because this is a small protein and not an antibody," she said. "Antibodies can have more side effects because they can form new complexes in the blood stream" that can either lead to unwanted activation of cytokines or to immune suppression that increases susceptibility to serious infections.

Liu added that Atsttrin should not induce unwanted immune reactions because it is derived from a natural protein.

Bachmann, however, thinks that Atsttrin has been so heavily modified that it could be more immunogenic than mAbs. He also noted that "there is really no reason to believe that the elevated risk of infection associated with other autoimmune disease treatments will be reduced by Atsttrin because, at the end of the day, you still block TNF."

Koh Ono, associate professor in the Department of Cardiovascular Medicine at Kyoto University, noted that "progranulin was first identified as a tumor growth factor. Therefore, Atsttrin or progranulin need to be carefully given to RA patients with cancer."

"There is a worry that progranulin is a growth factor, and the question of whether it can cause cancer or not has not been tested," Ding agreed. "But Atsttrin is a truncated form with the C terminal that is responsible for the growth factor effect removed, so the risk probably shouldn't be there anymore."
A final advantage that Atsttrin could have over the anti-TNF biologics is in cost of manufacturing. Liu told SciBX that Atsttrin is produced in bacteria, which he said should be cheaper than antibodies.

Su told SciBX that the currently available TNF blockers are complex molecules produced by mammalian cell culture via recombinant DNA technologies, which cost hundreds to thousands of dollars per gram of product to manufacture. $\mathrm{He}$ said that the manufacturing cost of Atsttrin-related compounds can be as low as $10 \%-20 \%$ of the cost of TNF blockers produced in animal cells.

According to Laurent Galibert, head of inflammation and metabolic disorders at Addex Pharmaceuticals Ltd., a key consequence of the high cost of production, and thus the high price tag for anti-TNF antibodies, is that the drugs "are not used as a first-line therapy even though from a purely scientific standpoint they should be."

Addex has a small molecule TNFR1 negative allosteric modulator in the discovery stage to treat Alzheimer's disease (AD), multiple sclerosis (MS), RA, psoriasis and depression.

Martz, L. SciBX 4(12); doi:10.1038/scibx.2011.328

Published online March 24, 2011

\section{REFERENCES}

1. Tang, W. et al. Science; published online March 10, 2011; doi:10.1126/science.1199214

Contact: Chuan-ju Liu, New York University School of Medicine, New York, N.Y.

e-mail: chuanju.liu@med.nyu.edu

2. Bateman, A. \& Bennett, H.P.J. Bioessays 31, 1245-1254 (2009)

3. Kessenbrock, K. et al. J. Clin. Invest. 118, 2438-2447 (2008)

4. Yin, F. et al. J. Exp. Med. 207, 117-128 (2010)

5. Feng, J.Q. et al. FASEB J. 24, 1879-1892 (2010)

6. Baker, M. et al. Nature 442, 916-919 (2006)

7. Cruts, M. et al. Nature 442, 920-924 (2006)

\section{COMPANIES AND INSTITUTIONS MENTIONED}

Addex Pharmaceuticals Ltd. (SIX:ADXN), Geneva, Switzerland Amgen Inc. (NASDAQ:AMGN), Thousand Oaks, Calif.

ATreaon, no location yet

Cytos Biotechnology AG (SIX:CYTN), Zurich, Switzerland

Cytovance Biologics LLC, Oklahoma City, Okla.

Kyoto University, Kyoto, Japan

McGill University, Montreal, Quebec, Canada

Nankai University, Tianjin, China

New York University School of Medicine, New York, N.Y.

Pfizer Inc. (NYSE:PFE), New York, N.Y.

Shandong University School of Medicine, Jinan, China

Takeda Pharmaceutical Co. Ltd. (Tokyo:4502), Osaka, Japan

Texas A\&M Health Science Center, Houston, Texas

Weill Cornell Medical College, New York., N.Y.

Yale School of Medicine, New Haven, Conn. 